Tropical Journal of Pharmaceutical Research April 2017; 16 (4): 919-924

ISSN: $1596-5996$ (print); 1596-9827 (electronic)

(C) Pharmacotherapy Group, Faculty of Pharmacy, University of Benin, Benin City, 300001 Nigeria.

All rights reserved.

Available online at http://www.tjpr.org

Original Research Article

http://dx.doi.org/10.4314/tjpr.v16i4.25

\title{
Enhancing contrast of magnetic resonance imaging in patients with liver cirrhosis: Conveyance times of Primovist in hepatobiliary system
}

\author{
Abdallah Ahmed Elbakkoush ${ }^{1}$, Anas Khaleel ${ }^{2}$, Suleman Atique ${ }^{1}$, Albakush Nura \\ Ahmed Mohamed ${ }^{3}$, Isatou Sowe ${ }^{1}$ and Chien-Tsai Liu ${ }^{1^{*}}$ \\ ${ }^{1}$ Graduate Institute of Biomedical Informatics, College of Medical Science and Technology, Taipei Medical University, Taipei \\ 11031, Taiwan, ${ }^{2}$ Institute of Biological Chemistry, Academia Sinica, 128 Academia Road, Section 2, Nankang District, Taipei \\ 11529, Taiwan, ${ }^{3}$ Department of Zoology, Faculty of Sciences, Misurata University, Libya
}

${ }^{*}$ For correspondence: Email: ctliu@tmu.edu.tw

Received: 19 April 2016

Revised accepted: 5 March 2017

\begin{abstract}
Purpose: To determine transit times for excretion of gadoxetic acid (Gd-EOB-DTPA), a recent magnetic resonance imaging (MRI) contrast agent, in hepatobiliary system of patients with liver cirrhosis.

Methods: Liver cirrhosis patients that underwent contrast MRI examination at Renai Hospital, Taipei City, Taiwan were included. The patients who have experienced contrast-enhanced abdominal MR examination after injection of $10 \mathrm{~mL}$ Gd-EOB-DTPA at 1.5-T MR from December 2009 to March 2011, were included retrospectively. The images were evaluated for the presence of contrast agent in intrahepatic bile ducts (IHD), common bile duct (CBD), gall bladder and duodenum.

Results: The optimal time for arterial phase was from $15 \mathrm{~s}$ after injection while the optimal time for portal venous imaging was from $40 \mathrm{~s}$ after injection. Furthermore, the optimal time to observe changes was 20 min after contrast initiation of Gd-EOB-DTPA in 39 patients (83\%) in IHD and 37 patients (78.5 $\%)$ in CBD. Gall bladder reflux was visible in 26 patients (43\%), and duodenal excretion in 17 patients (36\%). After 30 min of contrast injection, Gd-EOB-DTPA could still be detected in 6 patients (13\%) in IHD and 7 patients (15\%) in CBD, while gall bladder reflux was visible in 10 patients (21\%), and duodenal excretion in 20 patients (55\%).

Conclusion: The excretion of Gd-EOB-DTPA can be observed in liver cirrhosis patients.
\end{abstract}

Keywords: Hepatocellular carcinoma, Magnetic resonance imaging (MRI), Gadoxetic acid, Common hepatic duct

Tropical Journal of Pharmaceutical Research is indexed by Science Citation Index (SciSearch), Scopus, International Pharmaceutical Abstract, Chemical Abstracts, Embase, Index Copernicus, EBSCO, African Index Medicus, JournalSeek, Journal Citation Reports/Science Edition, Directory of Open Access Journals (DOAJ), African Journal Online, Bioline International, Open-J-Gate and Pharmacy Abstracts

\section{INTRODUCTION}

The liver is the most metabolically complex organ in human body. It is large and complex organ with diverse functions, many of them are critical for survival [1]. The approaches for medical diagnosis and disease characterization of the liver and the biliary system via non-invasive methods have greatlyimproved over the previous decades due to the unending development of computed tomography (CT), sonography or ultrasound (US), nuclear medicine (NM) and magnetic resonance imaging (MRI) [2]. Contrary to CT and NM, Magnetic Resonance imaging does not expose the patient to any ionizing radiation [3]. MRI of superior body soft-tissue contrast was believed to provide enough diagnostic information for most needs, without the use of contrast media [4]. Gadolinium-based and other contrast media were, however, soon 
considered to be of significant importance in disease detection and characterization [5].

Gd-EOB-DTPA (gadolinium ethoxybenzyl diethylenetriaminepentaacetic acid, Primovist ${ }^{\circledR} \quad 0.25$ $\mathrm{mmol} / \mathrm{ml}$, Bayer Schering Pharma, Berlin, Germany) is a more recently approved liverspecific contrast agent $[5,6]$. It combines hepatocellular specificity with T1-relaxivity and extracellular behavior [7]. After intravenous injection, Gd-EOB-DTPA is first distributed into the extracellular space and then taken up by the hepatocytes.

It is excreted un-metabolized form in equal proportions by the kidneys and ATP-dependent active transport in the hepatocytes to the biliary system. Due to which, the hepatobiliary excretory proportion is approximately ten times greater than for Gd-BOPTA. Renal excretion of Gd-EOBDTPA can be substituted by the hepatobiliary elimination and vice versa [8].

\section{METHODS}

\section{Study design}

This is a retrospective study analyzing data from the hospital database as the study material. We collected all the patients' baseline information including the Child Pugh scores, bilirubin level, and liver function. We followed guidelines given by American Association of study of liver diseases [22].

\section{Patient population}

Institutional Review Board of Taipei City Hospital - Renai Branch approved the study and informed consent was waived. Patients with liver cirrhosis but with normal glomerular filtrate rate (GFR) were included in the analysis. All the patients underwent Gd-EOB-DTPA contrast-enhanced abdominal MR examination at Taipei City Hospital-Renai Branch from January 2011 to August 2012. Dataset of patient with ages equal or greater than 20 years were included in the study as the safety and effectiveness of GdEOB-DTPA has not been established in pediatric patients.

A total of 47 patients were enrolled in this study. Clinical and demographic information of the patients in the present study is illustrated in Table 1.

Patients younger than 18 years or with abnormal glomerular filtration rate were excluded from the study. Also Patients who had any contraindication to MRI (cardiac pacemaker, ferromagnetic implants, etc.) were excluded as well.

Table 1: Patient demographic data and clinical information of the patients

\begin{tabular}{ll}
\hline Age range (mean age) & $22-80$ years (57.3years) \\
Sex (M/F) & $32 / 15$ \\
Total bilirubin & $2.11 \mathrm{mg} / \mathrm{dL}$ \\
AST/ALT & $46 / 40 \mathrm{IU} / \mathrm{L}$ \\
Creatinine & $0.98 \mathrm{mg} / \mathrm{dL}$ \\
CBD diameter & $4.84 \mathrm{~mm}(2.91-10.86)$ \\
Hepatitis $(\mathrm{n})$ & $\mathrm{B}(40)$ \\
& $\mathrm{C}(4)$ \\
& Alcoholic (2) \\
& B +Alcoholic (1) \\
Child-Pugh class (n) & A (40) \\
& B (5) \\
& C (2) \\
\hline
\end{tabular}

\section{Imaging Methods}

All MRI examinations of the liver of the enrolled patients were performed with Achieva 1.5T ASeries MRI Scanner from Philipsphased-array body coil. Prior to contrast agent administration, turbo spin-echo (TSE) T2WI (TR/TE: 1000 1800/110 milliseconds; Slice thickness 8mm; Gap $0.8 \mathrm{~mm}$; Matrix, 192×256; TSE factor, 24; number of average [NEX], 2; Flip angle $90^{\circ}$; FOV, $38-40 \mathrm{~cm}$ ) without and with fat saturation, and coronal T2WI were obtained under respiratory trigger. Dual T1WI (TR/TE: 180/2.3/4.6; Slices thickness 8mm; Matrix, 192×256; NEX, 1; Flip angle 10 ${ }^{\circ}$; FOV, 38-40 $\mathrm{cm}$ ) and fat sat T1WI were performed during one breath hold. Automatic shimming has been used for fat suppression imaging to maximize magnetic field homogeneity. Flow compensation is also used for the same purpose.

For the contrast-enhanced MRI, all the patients received a dose of $0.025 \mathrm{mmol} / \mathrm{kg}$ or $10 \mathrm{ml}$ of $\mathrm{Gd}$ - EOB-DTPA (Primovist; Bayer Schering Pharma, Osaka, Germany). The contrast agent was injected as a bolus at a speed of nearly 2 $\mathrm{ml} / \mathrm{s}$ through peripheral veins. Dynamic 3D T1weighted fast field echo (FFE) sequence (TR/TE: 5 - 10/3.3 ms; Slice thickness 5 mm; Matrix, 192 $\times 256$; NEX, 1; Flip angle $10^{\circ}$; FOV, $38-40 \mathrm{~cm}$ ) was carried out before, 18 - $20 \mathrm{~s}$ (arterial phase, AP), $50 \sim 55 \mathrm{~s}$ (portal venous phase, PP), $85 \sim$ $90 \mathrm{~s}$ (venous phase, VP) following the contrast agent injection. In addition, three delay phase images $(180 \mathrm{~s}, 20 \mathrm{~min}$ and $30 \mathrm{~min}$ after the injection of contrast agent) were acquired.

\section{Imaging analysis}

The images were evaluated for the presence of 
contrast agent in the intra-hepatic bile ducts (IHD), the common bile duct (CBD), the gallbladder and the duodenum in AP, PP, VP and three delayed phases. The images were evaluated by the same radiologist. He had to record the phase in which the Gadoxetic acid appeared.

\section{Statistical analysis}

The data has been presented as mean \pm standard deviation (SD). Each experiment was repeated at least 3 times. Statistical analysis was performed using SPSS version 19 for one-way analysis of variance (ANOVA) with $p<0.05$ being significant, was used to determine whether there was significant difference in time of first appearance of contrast agent in the individual segments of the hepatobiliary tract, with the cirrhotic degree (according to Child-Pugh score) and the bilirubin level (according to mean value of bilirubin and the level of bilirubin data).

\section{RESULTS}

The demographic patient data and clinical indications for the abdominal MR examinations are provided in Table 1. Out of the 47 patients, gall bladder (GB) was not visible in 9 patients, 4 out of 9 patients had cholecystectomy. The GB might have been removed due to complications associated with the gall bladder i.e. gall stones, and gall bladder cancer etc. The mean CBD diameter was $4.84 \mathrm{~mm}$.

The optimal time of arterial phase was from 18 $20 \mathrm{~s}$ after injection, while for the portals, $50-55 \mathrm{~s}$ after injection for the venous phase. The mean time for opacification was after 20 min contrast injection in 39 patients $(39 / 47,83 \%)$ at the IHD in 37 patients $(37 / 47,78 \%)$ at the CBD. Gall bladder reflux was visible in $26(26 / 47,43 \%)$ and 17 patients $(17 / 47,36 \%)$ at the duodenal. After $30 \mathrm{~min}, \mathrm{Gd}$ - EOB-DTPA could still be detected in 6 patients $(6 / 47,13 \%)$ at the IHD and in 7 patients $(7 / 47,15 \%)$ at the CBD. Gallbladder reflux was visible in 10 patients $(10 / 47,21 \%)$, duodenal excretion in 26 patients (26/47, $55 \%$ ) respectively as shown in Table 2.

On visualization of the biliary tree, the distribution was not statistically significant $(p<0.05)$ between bilirubin level and visualization of the biliary tree as shown on Table 3 .

On Visualization of Gd - EOB-DPTA contrast in IHD and CBD for Patients with different bilirubin level, the excretion of Primovist in the biliary tree is affected by the bilirubin level. After $30 \mathrm{~min}$, the $p$ value was significant $(p=0.043)$ in comparison with group 1 and group 3 . The $p$ value was not significant, between group 1 and $2(p=0.27)$ after $30 \mathrm{~min}$ of injection. The $p$-value was not statistically significant between group 2 and group 3, after 30 min post injection $(p=0.226)$ as provided in Table 4.

In group three, where the bilirubin level is more than $2 \mathrm{mg} / \mathrm{dl} 30$ minutes' post injection, the visualization level is $40 \%$ as shown in Figure 1. This illustrates the severity of liver cirrhosis can influence the uptake and excretion of the contrast agent. Dahlqvistet.al, demonstrated that impaired hepatobiliary function severely influences the hepatic uptake of Gd - EOB - DTPA when they did a study on 31 patients on how to quantify the hepatocyte specific uptake of Gd - BOPTA and $\mathrm{Gd}$ - EOB - DTPA using dynamic contrast enhanced (DCE) MRI [19].

Table 2: Transit times in various segments of the biliary excretion route

\begin{tabular}{|c|c|c|c|c|c|c|}
\hline $\begin{array}{l}\text { Biliary excretion } \\
\text { segment }\end{array}$ & AP & PP & VP & $3 \min$ & $20 \mathrm{~min}$ & 30min \\
\hline IHD & 0 & 0 & $\star 2 / 0 / 0$ & 0 & ${ }^{*} 36 / 3 / 0$ & ${ }^{\star} 2 / 2 / 2$ \\
\hline CBD & 0 & 0 & *3/0/0 & 0 & *34/3/0 & ${ }^{*} 3 / 2 / 2$ \\
\hline GB & 0 & 0 & * $1 / 0 / 0$ & 0 & *24/2/0 & ${ }^{*} 5 / 3 / 2$ \\
\hline Duodenum & 0 & 0 & 0 & * $1 / 0 / 0$ & * $17 / 0 / 0$ & *19/5/2 \\
\hline
\end{tabular}

Table 3: Bilirubin levels on visualization of the biliary tree

\begin{tabular}{lcccccccc}
\hline Phase & \multicolumn{2}{c}{ IHD } & \multicolumn{2}{c}{ CBD } & \multicolumn{2}{c}{ GB } & \multicolumn{2}{c}{ Duodenum } \\
\cline { 2 - 8 } & No. & Bilirubin & No. & Bilirubin & No. & Bilirubin & No. & Bilirubin \\
\cline { 2 - 8 } & 2 & $0.75 \pm 0.35$ & 3 & $0.79 \pm 0.26$ & 1 & 0.87 & 0 & VP \\
3 min & 0 & 0 & 0 & 0 & 0 & 0 & 1 & 0.87 \\
$20 \mathrm{~min}$ & 39 & $2.07 \pm 5,59$ & 37 & $2.13 \pm 5.74$ & 26 & $2.44 \pm 6.86$ & 17 & $3.11 \pm 8.48$ \\
$30 \mathrm{~min}$ & 6 & $2.85 \pm 3.69$ & 7 & $2.58 \pm 3.44$ & 10 & $2.16 \pm 2.91$ & 26 & $1.57 \pm 1.85$ \\
$P$-value & & 0.885 & & 0.888 & & 0.964 & 0.653 \\
\hline
\end{tabular}


No. indicates number of patients

Table 4: Visualization of Gd - EOB-DPTA contrast in IHD and CBD for patients with different bilirubin level

\begin{tabular}{|c|c|c|c|c|}
\hline Variable & & $\begin{array}{l}\text { Group } 1(27) \\
(>1.2 \mathrm{mg} / \mathrm{dl})\end{array}$ & $\begin{array}{c}\text { Group } 2(15) \\
(1.2-2.1 \mathrm{mg} / \mathrm{dl})\end{array}$ & $\begin{array}{l}\text { Group } 3(5) \\
\text { (>2 } \mathrm{mg} / \mathrm{dl})\end{array}$ \\
\hline \multirow[t]{2}{*}{ Venous phase } & IHD & 2 & - & - \\
\hline & CBD & 3 & - & - \\
\hline \multirow[t]{2}{*}{$3 \mathrm{~min}$} & IHD & - & - & - \\
\hline & CBD & - & - & - \\
\hline \multirow[t]{2}{*}{$20 \mathrm{~min}$} & IHD & 23 & 12 & 3 \\
\hline & CBD & 21 & 12 & 3 \\
\hline $30 \mathrm{~min}$ & $\begin{array}{l}\text { IHD } \\
\text { CBD }\end{array}$ & $\begin{array}{l}2(2 / 27) 7.4 \% \\
3(3 / 270) 11 \%\end{array}$ & $\begin{array}{l}3(3 / 15) 20 \% \\
3(3 / 15) 20 \%\end{array}$ & $\begin{array}{l}2(2 / 5) 40 \% \\
2(2 / 5) 40 \%\end{array}$ \\
\hline
\end{tabular}

(a)

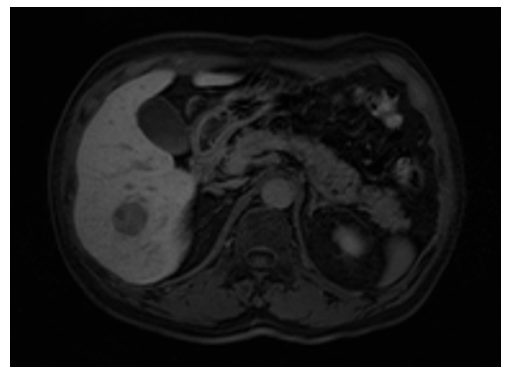

(b)

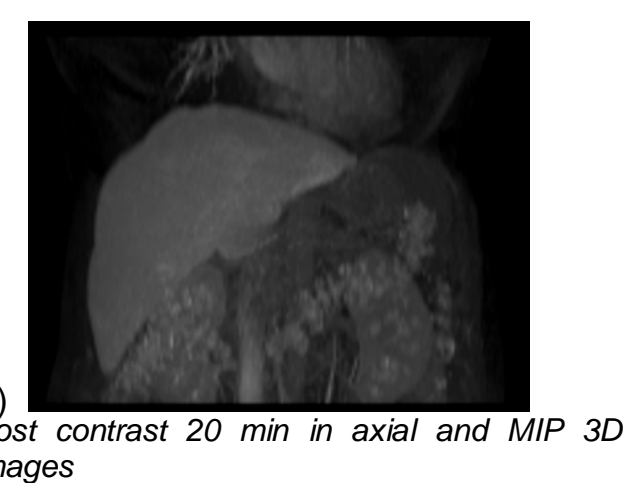

(c)

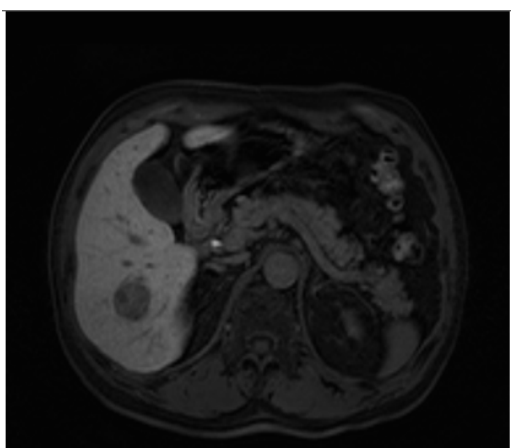

(d)

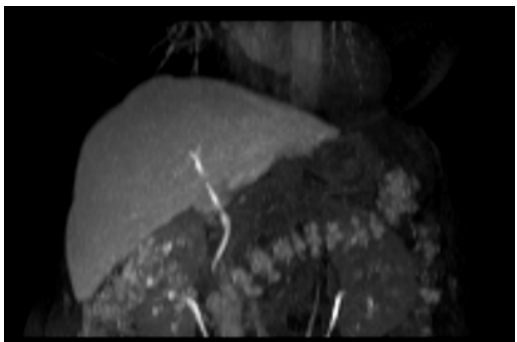

Post contrast 30 min in axial and MIP 3D images

Figure 1: Appearance of Gd-EOB-DTPA in the hepatobiliary System after 20 and 30 min in Subfigure $\mathrm{a}, \mathrm{b}, \mathrm{c}$ and $\mathrm{d}$

\section{DISCUSSION}

Currently, data regarding transit times of $\mathrm{Gd}$ EOB-DTPA via the biliary excretion route in the patients is limited, as most of the 'delayed imaging time course data' have been gathered from healthy volunteers. Our analysis which was performed on the patients with liver cirrhosis showed no difference in terms of transit times when compared to the other studies conducted on patients with normal liver. Kristina I. Ringe et al evaluated the hepatobiliary transit times of GdEOB-DTPA in 61 patients with normal liver function [14]. After $20 \mathrm{~min}$ of contrast initiation, Gd-EOB-DTPA was visible in the IHD and CBD in all patients.

In our study, 40 out of 47 patient were Child Pugh A. This might be the reason that there is no difference between our study and Kristina's study. In a phase I clinical evaluation, Hamm et al detected enhancement of the CBD as early as 10 min after contrast administration in 44 healthy volunteers [10]. Dahlström et al, who also evaluated the biliary excretion of Gd-EOB-DTPA in healthy subjects, later on confirmed these findings [15]. Bollow et al investigated the time course of contrast enhancement in the bile ducts in 16 healthy volunteers at 4 different doses of Gd-EOB-DTPA. Initial enhancement of the CBD was seen at a mean time of $10 \pm 4 \mathrm{~min}$ post contrast [11]. Our results are in line with the previously reported transit times for the intrahepatic bile ducts and the CBD but in patients with normal liver function which basically means that Gd-EOB-DTPA is not affected by liver disease. The number of patients in our study is small and the majority of the patients represented were only mildly affected by the disease. Patients with child Pugh $\mathrm{C}$ seemed to influence liver excretion of the contrast media. This confirms previous studies which indicate a tendency towards decreased diagnostic capability with the severity of cirrhosis.

Frank et al compared the quality of biliary duct 
visualization using Gd-EOB-DTPA enhanced magnetic resonance cholangiography in 40 adults with liver cirrhosis and 20 adults with normal liver parenchyma [18]. The overall quality was rated as sufficient for anatomical visualization of the biliary tree in all individuals of the control group 20 min after Gd-EOB-DTPA administration, but in only $16 / 40$ patients (40 \%) of the cirrhosis group within $30 \mathrm{~min}$ after administration. Analysis revealed non-sufficient visualization of the biliary tree $20 \mathrm{~min}$ after $\mathrm{Gd}$ EOB-DTPA administration, were total serum bilirubin levels $\geq 30 \mu \mathrm{mol} / \mathrm{l}$.

The excretion of Primovist in the biliary tree is affected by bilirubin level in group 3 where the bilirubin level is more than $2 \mathrm{mg} / \mathrm{dl} 30$ minutes post injection. The visualization level is 40 percent. This illustrates that the severity of liver cirrhosis can influence the uptake and excretion of the contrast agent. Dahlqvist et al demonstrated that impaired hepatobiliary function severely influences the hepatic uptake of Gd-EOB-DTPA when they did a study on 31 patients on how to quantify the hepatocytespecific uptake of Gd-BOPTA and Gd-EOBDTPA using dynamic contrast-enhanced (DCE) MRI [19].

Primovist can be detected in most of the patients after 20 minutes with child Pugh $A / B / C$ in this study. In the venous phase we can detect Primovist in patients $2 / 3 / 1, I H D, C B D, G B$ respectively early in the phase. It is important to note that all these patients have child Pugh $A$. Patients with child Pugh B and C seemed to influence the liver excretion of the contrast media. Our results were pretty similar to a study by Tsutomu Tamada that showed similar results after the MR image obtained in an 80-year-old woman with cirrhosis (Child-Pugh $\mathrm{C}$ ) acquired 20 min after Gd-EOB-DTPA administration[20,21].

\section{Study limitations}

One weakness of this study is the different distribution of the number of the study population in Child-Pugh class (A, B and $C$ ) with a high number of population in $(A)$ compared with very low population number in ( $B$ and $C$ ). This may have led to failure in having an insignificant $p$ value. As this was a retrospective study, the exact point of time for acquisition of the delayed images was not standardized.

\section{CONCLUSION}

It can be concluded that liver cirrhosis patients show no significant changes with regards to transit times when compared to normal patients.
Despite the difficulties in quantification of liver function, GD - EOB- DTPA enhanced MR imaging (MRI) offers a unique opportunity to combine qualitative and quantitative morphological and functional information that may improve the assessment of focal liver lesions and diffuse liver diseases and probe liver function.

\section{DECLARATIONS}

\section{Acknowledgement}

This study was supported by National Yang Ming University. Special thanks to Lin Joe and Prof Ran Chen Chiu for helping in data collection. The authors would also like to thank Renai Hospital for guidance in the study.

\section{Conflict of Interest}

No conflict of interest associated with this work.

\section{Contribution of Authors}

The authors declare that this work was done by the authors named in this article and all liabilities pertaining to claims relating to the content of this article will be borne by them.

\section{Open Access}

This is an Open Access article that uses a funding model which does not charge readers or their institutions for access and distributed under the terms of the Creative Commons Attribution License (http://creativecommons.org/licenses/by/ 4.0) and the Budapest Open Access Initiative (http://www.budapestopenaccessinitiative.org/rea d), which permit unrestricted use, distribution, and reproduction in any medium, provided the original work is properly credited.

\section{REFERENCES}

1. Debnath CR, Debnath MR, Alam MM, Moshwan MM, Alam MJ, Rana MS, Biswas D, Mahmuduzzaman M, Tarafder AJ. Cirrhosis of liver and portal vein thrombosis - A review article. Mymensingh Med J 2014; 606-608.

2. Arif-Tiwari H, Kalb B, Chundru S, Sharma P, Costello J, Guessner WR, Diego RM. MRI of hepatocellular carcinoma: an update of current practices. DiagnIntervRadiol2014;20: 209.

3. Surya C, Kalb B, Arif-Tiwari H, Sharma P, Costello J, Martin DR. MRI of diffuse liver disease: the common and uncommon etiologies. Diagn Interv Radiol2013;19: 479 
4. Peter $A B$. Twenty years of functional MRI: the science and the stories. Neuroimage Clin 2012; 62: 575-588.

5. Wanwarang $T$, Saiviroonporn $P$, Pongpaibul $A$, Korpraphong P. Benefit of double contrast MRI in diagnosis of hepatocellular carcinoma in patients with chronic liver diseases. I Med Assoc Thai. Chotmaihetthangphaet 2014; 97: 540-547.

6. Henrik N, Blomqvist L, Douglas L, Nordell A, Jonas E. Assessment of liver function in primary biliary cirrhosis using Gd-EOB-DTPA-enhanced liver MRI. HPB 2010; 12: $567-576$.

7. Janice W, Guthrie JA, Scott DJ, Atchley J, Wilson D, Davies MH, Wyatt JI, Robinson PJ. Hepatocellular carcinoma in the cirrhotic liver: double-contrast $M R$ imaging for diagnosis. Radiology 2000; 216: 154-162.

8. Simone $M$, Mainenti MP, Tambasco A, Imbriaco $M$, Mollica C, Laccetti E, Camera L, Liuzzi R, Salvatore $M$. Diagnostic accuracy of MR imaging to identify and characterize focal liver lesions: comparison between gadolinium and superparamagnetic iron oxide contrast media. Quant Imaging Med Surg 2014: 181-189.

9. Shao-Pow L, Brown JJ. MR contrast agents: Physical and pharmacologic basics. J Magn Reson Imaging2007; 25: 884-899.

10. Bernd H, Staks T, Mühler A, Bollow M, Taupitz M, Frenzel T, Wolf KJ, Weinmann HJ, Lange L. Phase I clinical evaluation of Gd-EOB-DTPA as a hepatobiliary $M R$ contrast agent: safety, pharmacokinetics, and MR imaging. Radiology 1995; 195: 785-792.

11. Bollow M, Taupitz M, Hamm B, Staks $T$, Wolf $K J$, Weinmann HJ. Gadolinium-ethoxybenzyl-DTPA as a hepatobiliary contrast agent for use in $M R$ cholangiography: results of an in vivo phase-I clinical evaluation. Eur. Radiol 1997; 7: 126-132.

12. Chia-Hsuin C, Lin JW, Wu LC, Liu CH, Lai MS. National antiviral treatment program and the incidence of hepatocellular carcinoma and associated mortality in Taiwan: a preliminary report. Med Care 2013; 51: 908913.

13. Giun-Yi H, Horng JL, Lee YS, Yen HJ, Chen CC, Lee CY. Cancer incidence patterns among children and adolescents in Taiwan from 1995 to 2009: A population-based study. Cancer 2014; 120: 3545-3553.

14. Kristina IR, Husarik DB, Rajan TG, Daniel TB, Elmar MM. Hepatobiliary transit times of gadoxetate disodium (Primovist $\Theta$ ) for protocol optimization of comprehensive MR imaging of the biliary system-what is normal? Eur J Radiol 2011; 79: 201-205.

15. Nils D, Persson A, Albiin N, Smedby O, Brismar TB. Contrast-enhanced magnetic resonance cholangiography with Gd-BOPTA and Gd-EOB-DTPA in healthy subjects. Acta Radiol 2007; 48: 362-368.

16. Ruth CC, Hero KH, Julie HS, Isaac RF. Gadoliniumethoxybenzyl-diethylenetriaminepentaacetic acid as an intrabiliary contrast agent: preliminary assessment. AJR Am J Roentgenol 2002; 179: 87-92.

17. Hogan WJ, Geenen JE. Biliary dyskinesia. Endoscopy 1988; 20: 179-183.

18. Frank TC, Struwe A, Petrowsky H, Kakales I, Marincek B, Weishaupt $D$. Contrast-enhanced MR cholangiography with Gd-EOB-DTPA in patients with liver cirrhosis: visualization of the biliary ducts in comparison with patients with normal liver parenchyma. Eur Radiol 2008; 18: 1577-1586.

19. Dahlqvist OL, Dahlström N, Kihlberg J, Sandström P, Brismar TB, Smedby O, Lundberg P. Quantifying differences in hepatic uptake of the liver specific contrast agents Gd-EOB-DTPA and Gd-BOPTA: a pilot study. Eur Radiol 2012; 22: 642-653.

20. Tsutomu $T$, Ito $K$, Sone $T$, Kanki $A$, Sato $T$, Higashi $H$. Gd-EOB-DTPA enhanced MR imaging: Evaluation of biliary and renal excretion in normal and cirrhotic livers. Eur J Radiol 2011; 80: e207-e211.

21. Dennis FW, Mallisee TA, Hohenwalter MD, Wilson CR, Quiroz FA, Taylor AJ. Multiphase hepatic CT with a multirow detector CT scanner. AJR Am J Roentgenol 2000; 175: 679-685.

22. Bruix J, Sherman M. Practice Guidelines Committee, American Association for the Study of Liver Diseases. Management of hepatocellular carcinoma. Hepatology 2005; 42: 1208-1236 